\title{
Lauroyl-L-aspartate decreased food intake and body temperature in neonatal chicks
}

\author{
E. Erwan ${ }^{\text {a }}$, V.S. Chowdhury ${ }^{\text {b }}$, K. Ito ${ }^{\text {a }}$, M. Furuse ${ }^{\text {a,* }}$, \\ a Laboratory of Regulation in Metabolism and Behavior, Graduate School of Bioresource and Bioenvironmental Sciences, Kyushu University, Fukuoka 812-8581, Japan \\ ${ }^{\mathrm{b}}$ Division for Arts and Science, Faculty of Arts and Science, Kyushu University, Fukuoka 819-0395, Japan
}

\section{A R T I C L E I N F O}

\section{Article history:}

Received 22 July 2013

Received in revised form 30 September 2013

Accepted 10 October 2013

Available online 17 October 2013

\section{Keywords:}

Lauroyl-L- or -D-aspartate

Body temperature

Food intake

\begin{abstract}
A B S T R A C T
We hypothesized that the effects of L- and D-amino acids might be influenced when conjugated with fatty acid. Thus, the effects of oral administration of lauroyl-L-aspartate (Lau-L-Asp) as well as lauroyl-D-aspartate (Lau-D-Asp) were examined. In Experiment 1, oral administration of both Lau-L-Asp and Lau-D-Asp decreased food intake while L- or D-Asp did not influence food intake. Interestingly, only Lau-L-Asp decreased body temperature. Experiment 2 was conducted to determine whether non-conjugated mixture of L-Asp plus lauric acid has same effects under ad libitum feeding conditions. Lau-L-Asp decreased food intake and body temperature, but L-Asp plus lauric acid did not show any effect studied. In Experiment 3, we found that Lau-L-Asp declined food intake as well as time-dependently suppressed the body temperature in fasted chicks. However, L-Asp plus lauric acid did not show any effect. These results suggest that Lau-L-Asp may exert anorexigenic and hypothermic actions in chicks.
\end{abstract}

(c) 2013 Elsevier Inc. All rights reserved.

\section{Introduction}

The regulation of food intake in poultry has been a focus of research interest in recent decades (Kuenzel, 1994; Richards, 2003). It has been reported that, unlike mammals, glucose does not play a major role for inducing satiety in birds (Smith et al., 1975; Gentle, 1976). One of the alternatives for such a role is performed by lipids. It has been reported that certain fatty acids such as the lauric acid, a medium-chain fatty acid or its chemically conjugated product with amino acids such as ethyl sodium-lauroyl-L-arginate hydrochloride (LAE), sodium lauroyl glutamate are being developed as a novel preservative in selected foods (Schwartz et al., 1997; Ruckman et al., 2004; Hawkins et al., 2009). Evans and Lepkovsky (1932) fed glyceryllaurate to rats at a $60 \%$ level in a synthetic diet and concluded this drug as a nontoxic one. Similar effects were found when rats were fed with mono-, di-, or triacylglyceroles at high levels in a synthetic diet for 70 days (Mattson et al., 1951). The test compound attributed no histological evidence to toxicity in rats. However, Cave (1982) observed that substitution of corn oil by lauric acid decreased food intake in broiler chick at the high level $(30 \mathrm{~g} / \mathrm{kg}$ ) but not at the low level $(10 \mathrm{~g} / \mathrm{kg})$. The reason for these different responses is likely to be the level of lauric acid in the diet. Furthermore, Little et al. (2005) administered lauric acid to healthy man and concluded that it has no effect on appetite, namely hunger, desire to eat, fullness or prospective consumption.

\footnotetext{
* Corresponding author at: Laboratory of Regulation in Metabolism and Behavior, Faculty of Agriculture, Kyushu University, Fukuoka 812-8581, Japan. Tel.: +8192642 2953; fax: + 81926422954 .

E-mail address: furuse@brs.kyushu-u.ac.jp (M. Furuse).
}

Since there has been existing conflicts regarding the hazard of adding synthetic lauric acid-emulsifier to food, the studies on the effect of feeding lauric acid emulsifiers have been investigated more thoroughly. Harris et al. (1951a,b) studied the effects of feeding sorbitan monolaurate, polyoxyethylene monolaurate, and polyoxyethylene monostearate in rats at $25 \%$ of their diet and in hamsters at 5 and $15 \%$ levels. They reported severe diarrhea, decreased weight gains, and increased mortality at all feeding levels and noted pathological conditions in rats fed the lauric acid emulsifiers. In non-mammalian vertebrates including chickens, however, there was no study to investigate the effect of lauric emulsifier on food intake. As most of the lauric emulsifiers are anorexigenic in mammals, it is possible that such compound may also inhibit feeding behavior in chicks. As for chemical properties, while lauric acid is a powder, lauroyl-L-aspartate potassium salt (Lau-L-Asp) and lauroyl-D-aspartate potassium salt (Lau-D-Asp) are solutions where Lau-L-Asp contains 28\% solid at pH 7.7 and Lau-D-Asp contain $29 \%$ solid at pH 7.7. These solutions are new antimicrobial agents for using as cosmetic ingredients.

Several papers have discussed about the effect of amino acid supplementation on food intake or muscle metabolism. Asp occupies unique position in the intermediary metabolism, particularly in the mitochondria, where they play important roles in nitrogen and energy metabolism (Stegink, 1976). In avian species, similar to mammalian species, the L- and D-Asp have been shown to exist in the various brain regions of chickens (Neidle and Dunlop, 1990) and pigeon (Kera et al., 1996). It was reported that Asp is released from many tissues through non-classical neurotransmitter releasing pathways (D'Aniello, 2007; Hagberg et al., 1985; Homma, 2007), and in some cases can be enhanced by alterations of metabolism (Monda et al., 2003; 
Gundersen et al., 2001). It is well documented that brain neurochemical systems may regulate food intake, and a number of neurotransmitters contribute to this behavior. For example, intracerebroventricular (i.c.v.) administration or local administration of glutamate or the glutamate agonist, $N$-methyl-D-aspartate (NMDA), induced hyperphagia in mammals (Reddy et al., 1986; Ritter and Stone, 1987; Wandji et al., 1989).

Recently, we showed that oral administration of D-Asp but not L-Asp suppressed food intake in layer chicks (Erwan et al., 2013). Additionally, we demonstrated that central administration of both L- or D-Asp could induce a sedative effect, while the mechanism for hypnosis in neonatal chicks may be different for L-Asp in comparison with D-Asp (Erwan et al., 2012). Despite these existing evidences, it remains to be determined whether a chemical conjugate of L- or D-Asp with lipid, namely Lau-L-Asp or Lau-D-Asp could affect food intake. Moreover, recent reports on the presence of D-amino acids in diverse foods and the possibility of toxic effects of certain D-amino acids raises interest to investigate the effect of D-amino acids (Harper et al., 1970; Maruyama et al., 1972; Erwan et al., 2013). In this study, we therefore investigated the effect of Lau-L-Asp or Lau-D-Asp on food intake in chicks. Furthermore, we also investigated whether oral administration of these chemical conjugates affects body temperature of chicks to estimate the involvement of these chemicals in thermoregulation.

\section{Materials and methods}

\subsection{Animals and drugs}

One day-old layer chicks (Julia) were purchased from a local hatchery (Murata hatchery, Fukuoka, Japan) and housed in a wiremeshed cage $(50 \times 35 \times 33 \mathrm{~cm})$ in a group $(20-25$ chicks $)$ at a constant temperature of $30 \pm 1{ }^{\circ} \mathrm{C}$ and with continuous light. Chicks were all of the same age and were housed without any adult. Food (AX, Toyohashi Feed and Mills Co. Ltd., Aichi, Japan) and water were provided ad libitum. One day before the experiment, chicks (4 days old) were reared individually and assigned for treatment and control groups on the basis of their body weight in order to produce uniform groups. The number of animals used in each group was kept to the minimum $(n=5-8)$ that would still ensure adequate statistical power. This study was performed in accordance with the guidelines for animal experiments carried out in the Faculty of Agriculture and in the Graduate Course of Kyushu University, and adhered to Law no. 105 and Notification no. 6 of the government. L- and D-Asp were purchased from Wako Pure Chemical Industries (Osaka, Japan). Lau-L-Asp and Lau-D-Asp were supplied by Asahi KASEI, Kawasaki, Japan.

\subsection{Oral administration of the drugs}

Following an acclimatization period, chicks were randomly selected and divided into four groups. The chicks were reared individually in experimental cages and had ad libitum access to diet during the whole experimental period. On the day of the experiment, each chick (5 days old) was orally administered drug solutions by the elastic plastic needle on small syringe for treatment groups and distilled water for control group. Chicks received oral administration at a dose of $6 \mathrm{mmol} / \mathrm{kg}$ body weight of L-Asp for 120 min. The criterion of dose selection was based on our preliminary findings in which L-Asp and D-Asp (3.75 mmol $/ \mathrm{kg}$ body weight) caused no significant effect on food intake. Experiment 1 was conducted to examine whether oral administration of the treatments namely, L-Asp, D-Asp, Lau-L-Asp and Lau-D-Asp affects the food intake, and body temperature of chicks under an ad libitum feeding condition. The chicks were provided ad libitum diets during the experimental period for $2 \mathrm{~h}$ immediately after the treatment. Food intake (at 30, 60 and $120 \mathrm{~min}$ ) was determined by measuring the reduction in the amount of food consumed from a pre-weighed feeder. The weight of the feeder was measured using an electric digital balance.
At the end of the experiments, chicks were decapitated following anesthesia with isoflurane (Mylan Inc., Japan). Experiment 2 was conducted to determine whether any different effect occurs between the oral injection of the conjugate Lau-L-Asp and non-chemically bonded mixture of lauric acid plus L-Asp on food intake and body temperature under an ad libitum feeding condition. Experiment 3 was conducted similar to second experiment except chicks were fasted for $3 \mathrm{~h}$ prior to the drug administrations.

\subsection{Measurement of body temperature}

Body temperature of chicks was measured at 30,60 and 120 min after treatments using a digital thermometer with an accuracy of $\pm 0.1{ }^{\circ} \mathrm{C}$ (Thermalert TH-5, Physitemp Instruments Inc., USA), by inserting the thermistor probe in the cloaca to a depth of $1-2 \mathrm{~cm}$.

\subsection{Statistical analysis}

For food intake and body temperature parameters, a repeatedmeasures two-way ANOVA was applied. Significant differences were denoted as $P<0.05$. Values were presented as means \pm S.E.M. Statistical analysis was made using the commercially available package SAS (SAS Institute and Version 9.1. SAS Institute Inc., 2002). All data in each group were first subjected to a Thompson rejection test to eliminate outliers $(P<0.01)$, and the remaining data were used for the analysis among groups.

\section{Results}

3.1. Experiment 1: effects of oral administration of L- or D-Asp and Lau-L- or-D-Asp on food intake and body temperature

Fig. 1A shows the effects of oral administration of the drugs on food intake. Oral administration of L- or D-Asp did not affect food intake, while both Lau-L-Asp and Lau-D-Asp significantly $(F[4,32]=20.42$, $P<0.0001)$ reduced food intake and showed significant effect of the time $(F[2,8]=62.42, P<0.0001)$ and interaction between the treatment and time $(F[8,64]=8.54, P<0.0001)$, indicating that the Lau-L-Asp and Lau-D-Asp persistently reduced food intake with the progress of the experimental time. Fig. 1B shows the effect of oral administration of the drugs on body temperature during $2 \mathrm{~h}$ of the experimental period. A significant effect of Lau-L-Asp $(F[4,29]=11.07, P<0.0001)$ and time $(F[2,8]=4.35, P<0.05)$ were detected on body temperature. A significant interaction effect between Lau-L-Asp treatment and time was detected $(F[8,58]=4.26, P<0.001)$, indicating that the Lau-L-Asp persistently kept reduced body temperature with the advancement of time while reverse was true for the control chicks.

3.2. Experiment 2: effects of oral administration of L-Asp, lauric acid, lauric acid plus L-Asp and Lau-L-Asp on food intake and body temperature in ad libitum fed chicks

Fig. 2A shows the effects of oral administration of the drugs on food intake.

Oral administration of L-Asp, lauric acid or lauric acid plus L-Asp did not affect food intake, while Lau-L-Asp significantly $(F[4,30]=4.54$, $P<0.01)$ decreased food intake and also showed a significant effect of the time $(F[2,8]=93.53, P<0.0001)$ and interaction between the treatment and time $(F[8,60]=5.17, P<0.0001)$, implying that the effect of Lau-L-Asp, the time period of the experiment and their interaction caused constantly lower food intake. Fig. 2B shows the effect of oral administration of the drugs on body temperature during $2 \mathrm{~h}$ of the experimental period. Only Lau-L-Asp significantly decreased body temperature $(F[4,28]=12.25, P<0.0001)$. 

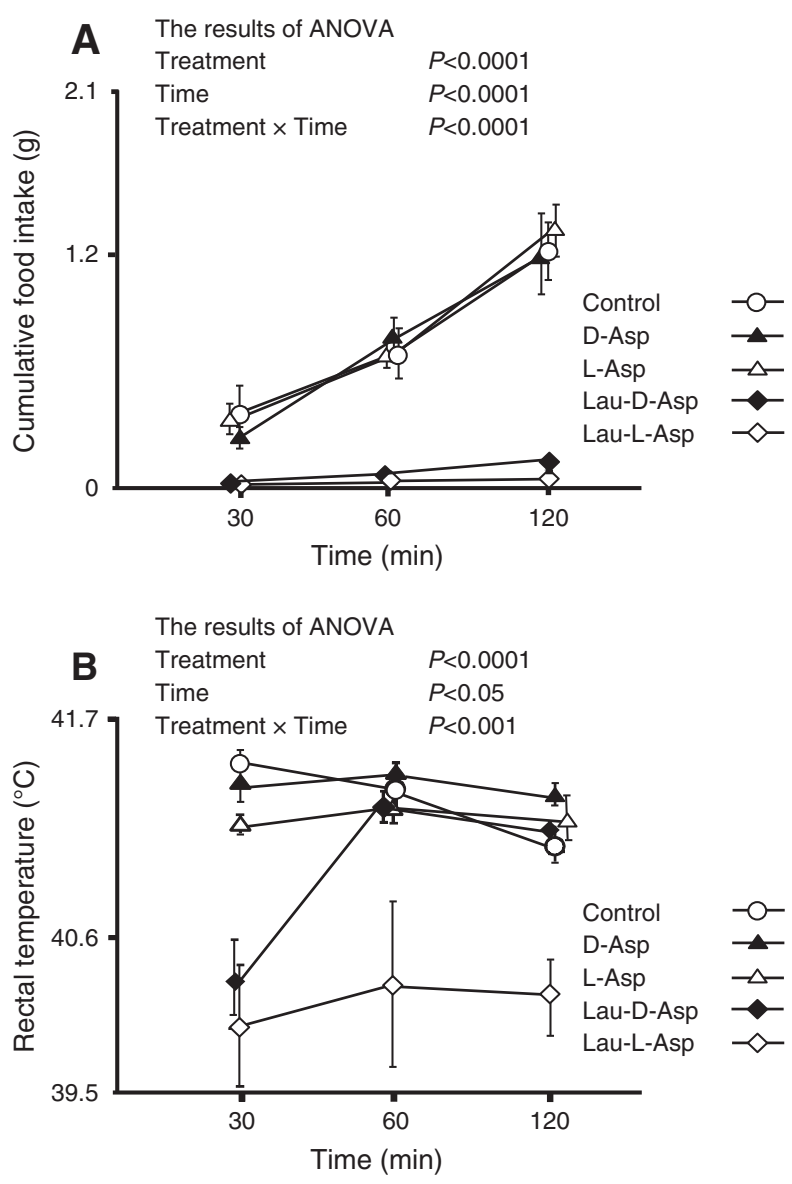

Fig. 1. The effects of oral administration of control, L-aspartate (L-Asp), D-aspartate (D-Asp), lauroyl-L-aspartate (Lau-L-Asp), lauroyl-D-aspartate (Lau-D-Asp) in chicks on food intake (A), and body temperature (B). The number of chicks used in each group ranged between 5 and 8 . Values are means \pm S.E.M.

3.3. Experiment 3: effects of oral administration of L-Asp, lauric acid, lauric acid plus L-Asp and Lau-L-Asp on food intake, and body temperature in fasted chicks

Fig. 3A shows the effects of oral administration of the drugs on food intake. While oral administration of L-Asp, lauric acid or lauric acid plus L-Asp did not affect food intake, Lau-L-Asp significantly decreased food intake $(F[4,27]=9.88, P<0.0001)$ and also showed significant effects of time $(F[2,8]=57.98, P<0.0001)$ and interaction effect between the treatment and time $(F[8,54]=3.48, P<0.005)$, denoting that the effect of Lau-L-Asp on food intake is interminable within the experimental time and their interaction effect also caused a reduced food intake while antithesis was valid for control group. Fig. 3B shows the effect of oral administration of the drugs on body temperature during $2 \mathrm{~h}$ of the experimental period. Only Lau-L-Asp significantly decreased body temperature $(F[4,27]=12.80, P<0.0001)$. Significant effects of time $(F[2,8]=7.39, P<0.005)$ and interaction $(F[8,54]=11.33$, $P<0.0001)$ between Lau-L-Asp and time were also detected, designating that the body temperature was kept lower in the Lau-L-Asp treated groups than in the control groups and it declined gradually with the progress of time in the treated groups.

\section{Discussion}

The present study clearly revealed that oral administration of both Lau-D-Asp and Lau-L-Asp (6 mmol/ $\mathrm{kg}$ ) consistently depressed food intake (Figs. 1A, 2A, 3A). However, only Lau-L-Asp decreased body
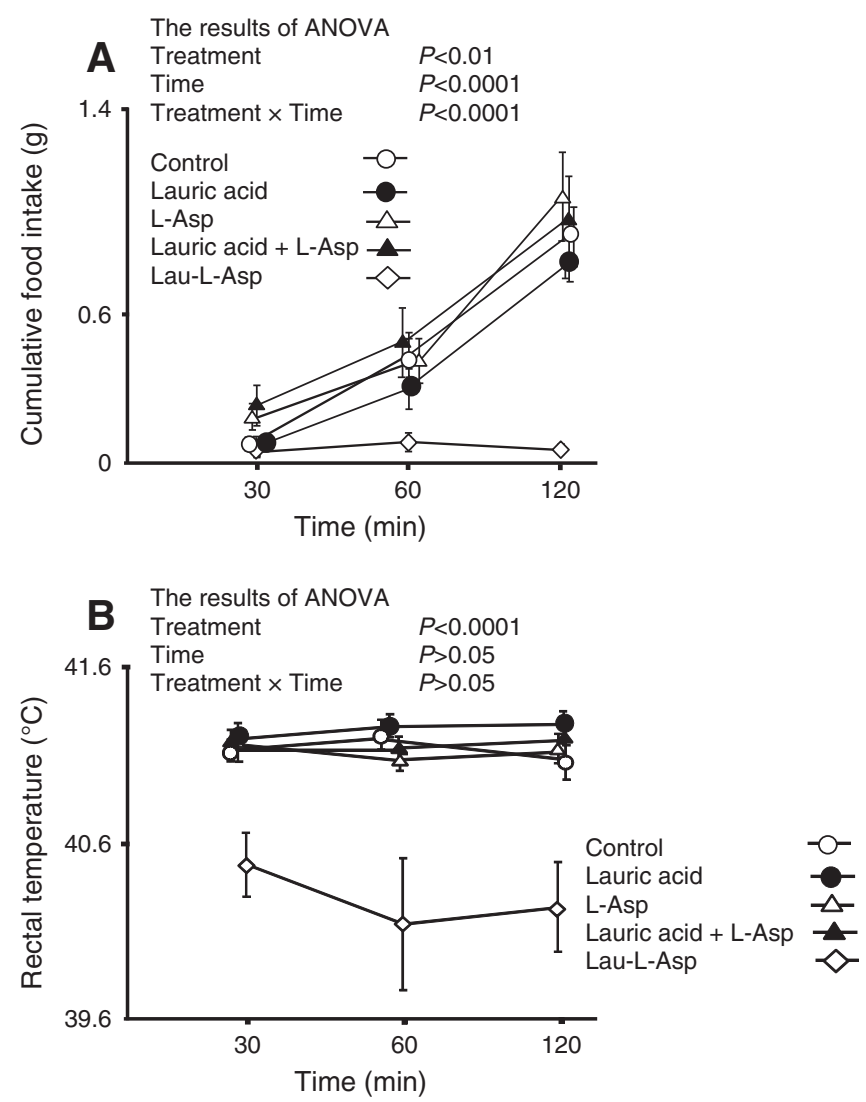

Fig. 2. The effects of oral administration of control, L-aspartate (L-Asp), lauric acid, lauric acid + L-Asp, lauroyl-L-aspartate (Lau-L-Asp) in ad libitum fed chicks on food intake (A), and body temperature (B). The number of chicks used in each group ranged between 5 and 8 . Values are means \pm S.E.M.

temperature. We previously revealed that there was no significant effect of D-Asp on food intake when orally administrated at the rate of $7.5 \mathrm{mmol} / \mathrm{kg}$ or lower than this amount, but at $15 \mathrm{mmol} / \mathrm{kg}$ showed a significant effect on food intake suppression (Erwan et al., 2013). Neither L-Asp nor D-Asp showed any effect on food intake in the current experiment which is inconsistent with our previous findings (Erwan et al., 2013). It is possible that the differences in dose caused this variation in response to food intake between the previous and present study. The dose of D-Asp used in the present study $(6 \mathrm{mmol} / \mathrm{kg}$ ) which was lower than those of the previous study $(15 \mathrm{mmol} / \mathrm{kg})$.

Since Lau-L-Asp and Lau-D-Asp caused to decrease food intake in the current study, it seems to be relevant with the following reports which indicate that lauric acid emulsifiers markedly reduced food consumption. For instance, Harris et al. (1951a,b) demonstrated that severe diarrhea, decreased weight gains, pathological conditions and increased mortality were occurred after feeding the emulsifiers of lauric acid in rats at $25 \%$ and in hamster at $5 \%$ and $15 \%$ of their diets. Krehl et al. (1955) concluded that there were low palatability of food when included polyeoxyethylene mono- and di-laurates and other polyoxyethylene esters in the diets of rats and cats. Eagle and Poling (1956) and Poling et al. (1956) revealed the decreased of growth rate and increased the mortality in rats, hamsters and rabbits fed polyoxythelene- 20 sorbitan mono-laurate from 5 to $25 \%$ of their feeding levels. Fitzhugh et al. (1960) demonstrated the decreasing body weight and toxicity in rats fed sorbitan mono-laurate (Span 20) and polyoxyethelene-20 monolaurate (G-2129) with lauric acid as emulsifiers. Ruckman et al. (2004) further observed that an LAE, lowered body weight and food consumption during the first week in rats when received 1143 and $1286 \mathrm{mg} / \mathrm{kg} / \mathrm{day}$. In light with the information mentioned above, it seemed that the reduced food intake 

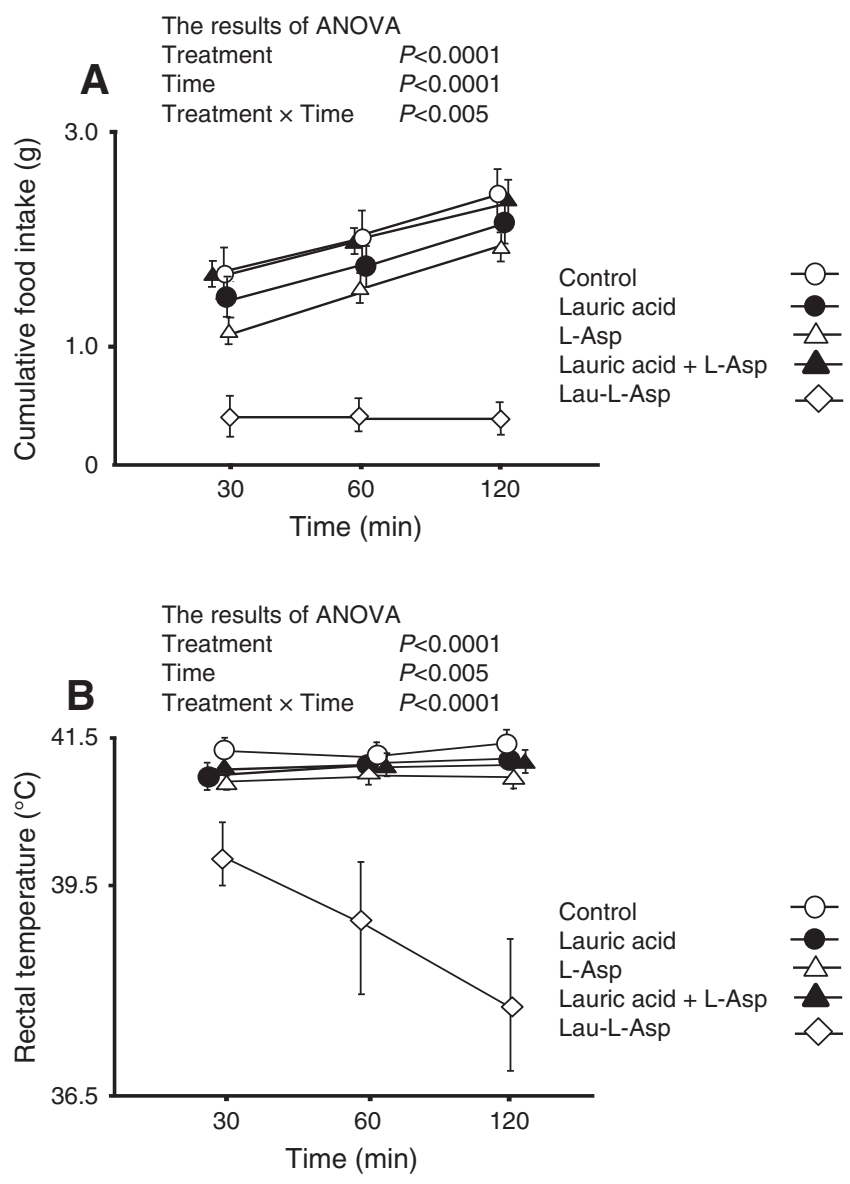

Fig. 3. The effects of oral administration of control, L-aspartate (L-Asp), lauric acid, lauric acid + L-Asp, lauroyl-L-aspartate (Lau-L-Asp) in fasted chicks on food intake (A), and body temperature (B). The number of chicks used in each group ranged between 5 and 8 . Values are means \pm S.E.M.

due to the oral administration of Lau-L-Asp or Lau-D-Asp could be explained, i.e., the anorexigenic effect of Lau-L-Asp and Lau D-Asp might be due to the emulsifier effect of lauric acid moiety with L-Asp or D-Asp.

To our knowledge, we are first to find out a fatty acid emulsifier that could be considered as a potential hypothermic agent. We have shown that oral administration of Lau-L-Asp, but not Lau-D-Asp, decreased body temperature (Fig. 1B). It seems to be an established fact that the metabolism and utilization of L- and D-Asp is different. For example, we revealed that there was a difference in the effect between L-Asp and D-Asp on stress response and food intake (Erwan et al., 2012, 2013) as well as on body temperature (unpublished data). These data raised the possibility of having different mechanism between the two isomers. In addition, the different effects on body temperature between Lau-L-Asp and Lau-D-Asp indicate that these two chemicals may work in different metabolic pathways for thermoregulation. Previous report revealed that the injection of D-Asp but not L-Asp released arginine vasotocin (AVT) in rats (Koyuncuoğlu et al., 1984). In addition, hypothermic actions of exogenous AVT were observed in sheep (Cooper et al., 1979; Ruwe et al., 1983) and rabbits (Naylor et al., 1985). Some reports have demonstrated that oral administration of DAsp decreased body temperature in rats (Koyuncuoğlu et al., 1982; Koyuncuoğlu and Berkman, 1982). Thus, we could assume that the chemical conjugation of D-Asp with lauric acid (Lau-D-Asp) inhibited its potential in reducing body temperature compared to Lau-L-Asp. Further study focusing on AVT release and thermoregulation in relation with Lau-D-Asp and Lau-L-Asp in chicks is necessary to understand the mechanism of actions of these drugs in thermoregulation. It is well known that the global surface temperature is increasing (IPCC, 2007) and high ambient temperature (HT) is a serious concern for birds as birds in general have a greater challenge than mammals in maintaining homeothermic body temperature (Ensminger et al., 1990; Chowdhury et al., 2012). Thus our current findings would help to think about some drug development to mitigate HT related problems.

We further examined separately the effect of the lauric acid, nonconjugated mixture of L-Asp plus lauric acid or conjugated Lau-L-Asp in Experiment 2. Neither L-Asp/lauric acid nor L-Asp plus lauric acid was associated with the decreasing food intake and body temperature. However, as observed in the Experiment 1, the form of Lau-L-Asp strongly depressed food intake and body temperature. Even though the doses of all treatments were similar but either oral administration of lauric acid or L-Asp plus lauric acid was not significant on food intake. These findings indicate that only when L- or D-Asp has emulsifier form; it may exhibit an anorexigenic effect. This result is consistent with the previous reports. Little et al. (2005) reported that there was no effect of lauric acid on appetite and food consumption in rats. Similar results revealed that there was no different digestibility in animals when fed either lauric acid or lauroyl glyceroles (Carrol, 1958; Deuel et al., 1941; Hoagland and Snider, 1943; Thomasson, 1956).

In Experiment 3, we confirmed more constant effects of Lau-L-Asp, on suppressing food intake and body temperature in fasted chicks (Fig. 3A,B). We cannot explain why oral administered Lau-L-Asp decreased body temperature so sharply in Experiment 3. However, we can speculate that Lau-L-Asp may play more effective role in thermoregulation in fasted chicks. Further experimentation is needed to clarify the mechanism.

In conclusion, orally administered Lau-L-Asp and Lau-D-Asp had a depressive effect on food intake in neonatal chicks. Importantly, it was demonstrated for the first time that Lau-L-Asp would be a potential emulsifier for food intake regulation and thermoregulation in chicks. Further investigations on Lau-L-Asp and Lau-D-Asp in controlling thermoregulation in chicks would be necessary to justify the possibility of utilizing the drug to mitigate HT related problems in chicks.

\section{Acknowledgments}

This work was supported by a Grant-in-Aid for Scientific Research (No. 23248046 to MF) from the Japan Society for the Promotion of Science. EE would like to thank the Directorate General of Higher Education (DGHE) of Indonesia for supporting a scholarship for this study.

\section{References}

Carrol KK. Digestibility of individual fatty acids in the rat. J Nutr 1958:64:399-410. Cave NAG. Effect of dietary short and medium chain fatty acids on feed intake by chicks. Poult Sci 1982;61:1147-53.

Chowdhury VS, Tomonaga S, Nishimura S, Tabata S, Cockrem JF, Tsutsui K, et al Hypothalamic gonadotropin-inhibitory hormone precursor mRNA is increased during depressed food intake in heat-exposed chicks. Comp Biochem Physiol A Mol Integr Physiol 2012;162:227-33.

Cooper KE, Kasting NW, Lederis K, Veale WL. Evidence supporting a role for endogenous vasopressin in natural suppression of fever in the sheep. J Physiol 1979;295:33-45.

D'Aniello A. D-Aspartic acid: an endogenous amino acid with an important neuroendocrine role. Brain Res Rev 2007;53:215-34.

Deuel HJ, Hallman JRL, Reifman A. The rate of absorption of various fatty acids by the rat. J Nutr 1941;21:373-82.

Eagle E, Poling CE. The oral toxicity and pathology of polyoxyethylene derivatives in rats and hamsters. J Food Sci 1956;21:348-61.

Ensminger ME, Oldfield JE, Heinemann WW. Feeds and nutrition. Clovis, CA: The Ensminger Publishing Company; 1990.

Erwan E, Tomonaga S, Yoshida J, Nagasawa M, Ogino Y, Denbow DM, et al. Central administration of L- and D-aspartate attenuates stress behaviors by social isolation and CRF in neonatal chicks. Amino Acids 2012;43:1969-76.

Erwan E, Tomonaga S, Ohmori T, Mutaguchi Y, Ohshima T, Nagasawa M, et al. Ora administration of D-aspartate, but not of L-aspartate, reduces food intake in chicks. J Poult Sci 2013;50:164-71.

Evans HM, Lepkovsky S. Vital need of the body for certain unsaturated fatty acids. II. Experiments with high fat diets in which saturated fatty acids furnish the sole source of energy. J Biol Chem 1932;96:157-64. 
Fitzhugh OG, Schouboe PJ, Nelson AA. Oral toxicities of lauric acid and certain lauric acid derivatives. Toxicol Appl Pharmacol 1960;2:59-67.

Gentle MJ. The effects of gold thioglucose on the central nervous system of chicks (Gallus domesticus). Toxicol Appl Pharmacol 1976;35:223-8.

Gundersen V, Fonnum F, Ottersen OP, Storm-Mathisen J. Redistribution of neuroactive amino acids in hippocampus and striatum during hypoglycemia: a quantitative immunogold study. J Cereb Blood Flow Metab 2001;21:41-51.

Hagberg H, Lehmann A, Sandberg M, Nystrom B, Jacobson I, Hamberger A. Ischemiainduced shift of inhibitory and excitatory amino acids from intra- to extracellular compartments. J Cereb Blood Flow Metab 1985;5:413-9.

Harper AE, Benevenga NJ, Wohlhueter RM. Effects of ingestion of disproportionate amounts of amino acids. Physiol Rev 1970;50:428-58.

Harris RS, Sherman H, Jetter WW. Nutritional and pathological effects of sorbitan monolaurate, polyoxyethylene sorbitan monolaurate, polyoxyethylene monolaurate and polyoxyethylene monostearate when fed torats. Arch Biochem Biophys 1951a;34:249-58.

Harris RS, Sherman H, Jetter WW. Nutritional and pathological effects of sorbitan monolaurate, polyoxyethylene sorbitan monolaurate, and polyoxyethylene monolaurate when fed to hamsters. Arch Biochem Biophys 1951b;34:259-65.

Hawkins DR, Rocabayera X, Ruckman S, Segret R, Shaw D. Metabolism and pharmacokinetics of ethyl N(alpha)-lauroyl-L-arginate hydrochloride in human volunteers. Food Chem Toxicol 2009;47:2711-5.

Hoagland R, Snider GG. Digestibility of certain higher saturated fatty acids and triglycerides. J Nutr 1943;26:219-25.

Homma H. Biochemistry of D-aspartate in mammalian cells. Amino Acids 2007;32: 3-11.

Intergovernmental Panel on Climate Change (IPCC). Climate change 2007: the physical science basis. Contribution of Working Group I to the Fourth Assessment Report of the Intergovernmental Panel on Climate Change. http://ipccwg.ucar. edu/wg/ Report/AR WG_Print_SPM.pdf, 2007.

Kera Y, Aoyama H, Watanabe N, Yamada RH. Distribution of D-aspartate oxidase and free D-glutamate and D-aspartate in chicken and pigeon tissues. Comp Biochem Physiol B Biochem Mol Biol 1996;115:121-6.

Koyuncuoğlu H, Berkman K. Effect of D- and/or L-aspartic acids on feeding, drinking, urine outflow and core temperature. Pharmacol Biochem Behav 1982;17: 1265-9.

Koyuncuoğlu H, Berkman K, Wildmann J, Matthaei H. Antagonistic effect of L-aspartic acid on decrease in body weight, and food and fluid intake, and naloxone reversible body temperature depression caused by D-aspartic acid. Pol J Pharmacol Pharm 1982;34: 333-7.

Koyuncuoğlu H, Berkman K, Hatipoğlu I, Sabuncu H. Vasopressin release by D-aspartic acid, morphine and prolyl-leucyl-glycinamide (PLG) in DI Brattleboro rats. Pharmacol Biochem Behav 1984;20:519-25.

Krehl WA, Cowgill GR, Whedon AD. Non-deleterious effects of polyoxyethylene esters in the nutrition of rats and cats. J Nutr 1955;55:35-61.
Kuenzel WJ. Central neuroanatomical systems involved in the regulation of food intake in birds and mammals. J Nutr 1994;124:355S-70S.

Little TJ, Feltrin KL, Horowitz M, Smout AJ, Rades T, Meyer JH, et al. Dose-related effects of lauric acid on antropyloroduodenal motility, gastrointestinal hormone release, appetite, and energy intake in healthy men. Am J Physiol Regul Integr Comp Physiol 2005;289:1090-8.

Maruyama K, Sunde ML, Harper AE. Effect of D-alanine and D-aspartic acid on the chick. J Nutr 1972;102:1441-51.

Mattson FH, Baur FJ, Beck LW. The comparative nutritive value of mono-, di- and triglycerides. J Am Oil Chem Soc 1951;28:386-90.

Monda M, Viggiano A, De Luca V. An aversive diet as thiamine-free food blocks food-induced release of excitatory amino acids in the accumbens. Acta Physiol Scand 2003;178:197-203.

Naylor AM, Ruwe WD, Kohut AF, Veale WL. Perfusion of vasopressin within the ventral septum of the rabbit suppresses endotoxin fever. Brain Res Bull 1985;15:209-13.

Neidle A, Dunlop DS. Developmental changes of free D-aspartic acid in the chicken embryo and in the neonatal rat. Life Sci 1990;46:1517-22.

Poling CE, Eagle E, Rice EE. Effects of feeding polyoxyethylene preparations to rats and hamsters. J Food Sci 1956;21:337-47.

Reddy VM, Meharg SS, Ritter S. Dose-related stimulation of feeding by systemic injections of monosodium glutamate. Physiol Behav 1986;38:465-9.

Richards MP. Genetic regulation of feed intake and energy balance in poultry. Poult Sci 2003;82:907-16.

Ritter S, Stone SL. Area postrema lesions block feeding induced by systemic injections of monosodium glutamate. Physiol Behav 1987;41:21-4.

Ruckman SA, Rocabayera X, Borzelleca JF, Sandusky CB. Toxicological and metabolic investigations of the safety of $\mathrm{N}$-a-Lauroyl-L-arginine ethyl ester monohydrochloride (LAE). Food Chem Toxicol 2004;42:245-59.

Ruwe WD, Veale WL, Cooper KE. Peptide neurohormones: their role in thermoregulation and fever. Can J Biochem Cell Biol 1983;61:579-93.

SAS Institute, version 9.1. SAS Institute Inc., Cary., NC, USA. 2002.

Schwartz S, Clare R, Devereux K, Sheung CF. Pharmacokinetics, disposition and metabolism of 546C88 (L-NG-methylarginine hydrochloride) in rat and dog. Xenobiotica 1997;27:1259-71

Smith CJ, Hatfield J, Fowler S, Bright-Taylor B. Changes in food consumption and blood glucose levels in the domestic chicken, Gallus domesticus, in response to the administration of 2-deoxy-D-glucose. Comp Biochem Physiol A Comp Physiol 1975;51:811-4.

Stegink LD. Absorption, utilization, and safety of aspartic acid. J Toxicol Environ Health 1976;2:215-42.

Thomasson HJ. The biological value of oils and fats. IV. The rate of intestinal absorption. J Nutr 1956;59:343-52.

Wandji SA, Seoane JR, Roberge AG, Bédard L, Thibault L. Effects of intrahypothalamic injections of GABA, muscimol, pentobarbital, and L-glutamic acid on feed intake of satiated sheep. Can J Physiol Pharmacol 1989;67:5-9. 